Pacific Journal of Mathematics

STUDYING LINKS VIA CLOSED BRAIDS. I: A FINITENESS JAN BIRMAN AND WilLIAM W. MENASCo 


\title{
STUDYING LINKS VIA CLOSED BRAIDS I: A FINITENESS THEOREM
}

\author{
Joan S. Birman and William W. Menasco
}

\begin{abstract}
This paper is the first in a series which study the closed braid representatives of an oriented link type $\mathscr{L}$ in oriented 3-space. A combinatorial symbol is introduced which determines an oriented spanning surface $F$ for a representative $L$ of $\mathscr{L}$. The surface $F$ is in a special position in 3-space relative to the braid axis $A$ and the fibers in a fibration of the complement of $A$. The symbol simultaneously describes $F$ as an embedded surface and $L$ as a closed braid. Therefore it is both geometrically and algebraically meaningful. Using it, a complexity function is introduced. It is proved that $\mathscr{L}$ is described by at most finitely many combinatorial symbols, and thus by finitely many conjugacy classes in each braid group $B_{n}$ when the complexity is minimal.
\end{abstract}

1. Introduction. This paper is the first in a series of papers in which the authors study the closed braid representatives of oriented links in oriented 3-space. Our goal is to develop a "calculus" for links in $S^{3}$. By this we mean a systematic procedure which begins with an arbitrary representative $\mathbf{L}$ of an arbitrary link type $\mathscr{L}=[\mathbf{L}]$, assigns to it an appropriate measure of complexity, detects when the complexity is not minimal and modifies $\mathbf{L}$ via a sequence of links of decreasing complexity to a canonical representative or a finite set of canonical representatives.

In this manuscript we will set up basic machinery which will be used throughout the series and will prove that our problem is solvable in the following sense: (i) we define an appropriate complexity function, and (ii) we prove that there are only finitely many conjugacy classes in $B_{\infty}$ which represent a given link type and have minimum complexity. However, there is more to it than that. The complexity function which we define will be seen to be related to rich and interesting combinatorics. Our plan in subsequent papers is to put the combinatorics described here (possibly augmented by the addition of further structure) to work to detect when the complexity is non-minimal and to discover complexity-reducing changes in $\mathbf{L}$. Additional results in the program which are complete at this writing are reported on in [B-M] (which presents an overview of the first six papers in the program) 
and [B-M, II] through [B-M, VI], which treat various special aspects. Further work is planned.

To set the stage for our work, we go back to the beginnings of knot theory, at the end of the 19th century, when the Scottish physicist P. G. Tait initiated a major experimental investigation [Ta] of knots. Tait's plan was to describe knots by their diagrams, i.e. planar projections in which the singularities are at most a finite number of transverse double points, with overcrossings and undercrossings distinguished from one-another pictorially as one distinguishes them for highways on a map. Clearly each such diagram may also be described by a combinatorial symbol. For example, one might use the sequence of under and over-crossings encountered, in order, as one travels along the knot. The minimum number $d(\mathscr{L})$ of crossing points for a given knot type $\mathscr{L}$ is a knot type invariant; also there are at most finitely many distinct diagrams for each fixed crossing number. The plan was to assign a partial ordering to knot types via crossing number, and then to attempt to distinguish the various types as they first occur in the list by experimental techniques, hoping in this way to uncover the underlying principles which determine knotting.

Any knot which has a diagram with $d$ crossings also has diagrams with $d^{\prime}$ crossings for every $d^{\prime} \geq d$. This shows that the success of a program such as the one undertaken by Tait depends upon the discovery of a finite set of moves which will allow one to reduce the crossing number of a representative of $\mathscr{L}$ to $d(\mathscr{L})$, without increasing it on the way. Tait and his coworkers failed to find such a set of moves, and their entire project was ultimately abandoned.

We propose to study knots and links by methods which have some resemblance to Tait's ideas, but which use a measure of complexity which is more meaningful both geometrically and algebraically than the crossing number. A link $\mathbf{L}$ in $\mathbb{R}^{3}$ is represented as a closed $n$ braid if there is a line $\mathbf{A}$ (think of $\mathbf{A}$ as the $z$ axis) in $\mathbb{R}^{3}-\mathbf{L}$ and a choice of a fibration $\mathbf{H}$ of the open cylinder $\mathbb{R}^{3}-\mathbf{A}$ by half-planes $\left\{\left(\mathbf{H}_{\theta} ; \theta \in[0,2 \pi]\right\}\right.$, such that every intersection of $\mathbf{L}$ with a fiber $\mathbf{H}_{\theta}$ of $\mathbf{H}$ is transverse. See Figure 1a for an example. The integer $n$ is the number of points in $\mathbf{L} \cap \mathbf{H}_{\theta}$. It is independent of $\theta$ because intersections of $\mathbf{L}$ with $\mathbf{H}_{\theta}$ are transverse for every $\theta$. It was proved by Alexander [A1] that every link type $\mathscr{L}$ may be represented (in many ways) as a closed $n$-braid, for some $n$. The choice of a braid axis places geometric structure on $\mathbb{R}^{3}-\mathbf{A}$, in the form of the fibration $\mathbf{H}$, and we will put that structure to use. 


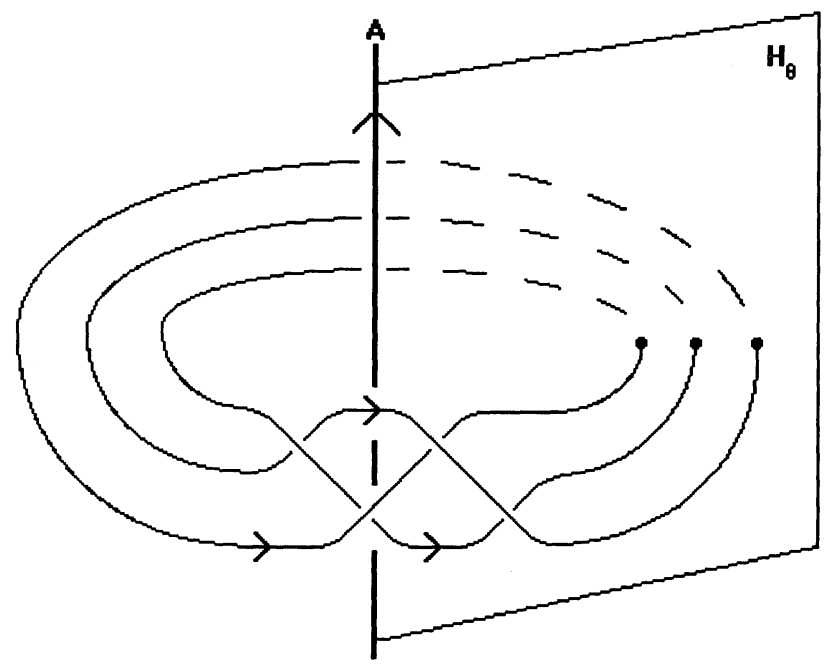

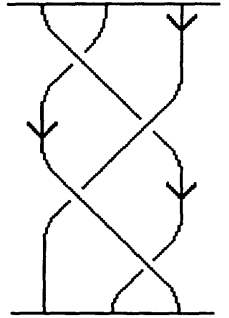

(b) open 3-braid representative

(a) closed 3-braid representative of the Figure 8 knot

Figure 1

In effect, the choice of an axis replaces the link type [L] by the collection of link types $[\mathbf{L}, \mathbf{A}]$, where $\mathbf{A}$ is an axis for an $n$-braid structure on $\mathbf{L}$, for some integer $n$. The choice of $\mathbf{A}$ and an associated set of half-planes $\mathbf{H}_{\theta}$ is essentially a choice of a coordinate system in 3-space.

There is also algebraic structure associated to closed braid representations of knots and links. To explain it, we first pass to the related concept of an open $n$-braid, illustrated by an example in Figure $1 \mathrm{~b}$. An open braid is obtained from a closed braid by cutting open 3space $\mathbb{R}^{3}-\mathbf{A}$ along a half-plane $\mathbf{H}_{\theta}$. The equivalence relation on open braids is the obvious one corresponding to isotopy of strings with fixed endpoints in $D^{2} \times 1$. One may multiply two open braids by juxtaposing them and concatenating them, and this makes them into a group, the $n$-string braid group $B_{n}$. The family of groups $\left\{B_{n}, n=1,2,3, \ldots\right\}$ has been studied extensively in the literature. See [Ar] for the key initial paper and [Bi] to get started with subsequent literature. Let $B_{\infty}$ denote the disjoint union of all of the braid groups $\left\{B_{n}, n=1,2,3, \ldots\right\}$. Call two elements of $B_{\infty}$ Markovequivalent [Ma] if the associated closed braids determine the same link type. By definition, Markov equivalence classes in $B_{\infty}$ are in 1-1 correspondence with oriented link types in oriented 3-space. 
It was proved by Morton in [Mo] that there is a one-to-one correspondence between conjugacy classes in $B_{n}, n=1,2,3 \ldots$, and oriented link types $[\mathbf{L}, \mathbf{A}]$, where $\mathbf{L}$ is a representative of $\mathscr{L}$ and $\mathbf{A}$ is a braid axis for $\mathbf{L}$. Thus the Markov class of $\mathscr{L}$ in $B_{\infty}$ is a union of conjugacy classes in $B_{\infty}$. Also, we may identify $[\mathbf{L}, \mathbf{A}]$ with a conjugacy class in some $B_{n}$. This brings us to an important fact: there are known solutions to the conjugacy problem in $B_{n}$. One such is due to Garside [G], and another is implicit in the work of Thurston [Th]. This means that a small part of the work of selecting a canonical representative for a link type has been done, as one may select a unique representative within each conjugacy class. For example, one might replace the infinitely many representatives of $[\mathbf{L}, \mathbf{A}]$ by the unique representative found by Garside's work [G]. However, we will not do that, as Garside's work does not seem to us to be well-adapted to geometry.

Returning to geometry, we note that an important tool was introduced by $\mathrm{D}$. Bennequin in [Be], in connection with his studies of contact structures on $\mathbb{R}^{3}$. Let $\mathscr{L}, \mathbf{L}, \mathbf{A}, \mathbf{H}$ be as above. Bennequin chooses an oriented spanning surface $\mathbf{F}$ for $\mathbf{L}$ which has maximal Euler characteristic, among all oriented spanning surfaces. He shows that $\mathbf{F}$ may be put into a nice position relative to the fibers of $\mathbf{H}$. He calls this modified surface F a "Markov surface" (relative to $\mathbf{H}$ ) for $\mathbf{L}$. The salient feature of his Markov surfaces is that there is a natural foliation of $\mathbf{F}$ by its arcs of intersection with fibers of $\mathbf{H}$, and the combinatorics of that foliation are rich and interesting. As we will see, they contain not only a finite set of data which determines $\mathbf{F}$ as an embedded surface relative to $\mathbf{H}$, even more they give instructions for the systematic simplification of $\mathbf{F}$ and/or $\mathbf{H}$, changing the braid representation but preserving the Euler characteristic of $\mathbf{F}$ and the link type of $\mathbf{L}=\partial \mathbf{F}$. Our basic idea is to put these combinatorics to work, in the service of the link problem. This approach of using spanning surfaces has been featured in a number of other studies concerning closed braids. For example, in [Mo3] spanning discs are used in the study of a special class of exchangable braids. Also, spanning surfaces were used in [R].

Here is our approach to the link problem. We begin with an arbitrary representative of $\mathscr{L}$. We add structure to this representative by choosing an axis $\mathbf{A}$ such that $\mathbf{L}$ is a closed braid representative of $\mathbf{L}$ with respect to $\mathbf{A}$. The equivalence class $[\mathbf{L}, \mathbf{A}]$ of the pair $(\mathbf{L}, \mathbf{A})$, under isotopy in 3-space then corresponds precisely to a conjugacy class of open braids in some braid group $B_{n}$ representing $\mathscr{L}$. We 
then add still more structure, replacing the equivalence class $[\mathbf{L}, \mathbf{A}]$ by an equivalence class $[\mathbf{F}, \mathbf{H}]$, where $\mathbf{F}$ is a surface of maximal Euler characteristic with $\mathbf{L}=\partial \mathbf{F}$, also $\mathbf{H}$ is a fibration of $\mathbb{R}^{3}-\mathbf{A}$, with $\mathbf{A}=\partial \mathbf{H}_{\theta}$. The equivalence relation on $(\mathbf{F}, \mathbf{H})$, which is described below, is chosen so that $[\mathbf{F}, \mathbf{H}]$ also determines a unique conjugacy class in some $B_{n}$. We prove in Theorem 1 that the surface $\mathbf{F}$ is determined, as an embedded surface relative to any convenient choice of $\mathbf{H}$, by a finite set of combinatorial data. This data replaces Garside's unique representative of the conjugacy class by one which is geometrically more meaningful. The first result in this paper (Theorem 1 below) proves that for any choice of an axis $\mathbf{A}$ and a fibration $\mathbf{H}$ of $\mathbb{R}^{3}-\mathbf{A}$ we may construct $\mathbf{F}$ as an embedded surface, relative to $\mathbf{H}$, from the combinatorial data.

We also introduce a complexity function on the collection $\{[\mathbf{F}, \mathbf{H}]\}$ of all equivalence classes $[\mathbf{F}, \mathbf{H}]$ associated to conjugacy classes of closed braids representing $\mathscr{L}$. The complexity function is related to the combinatorial data. The "finiteness theorem" of the title (see Theorem 2 below) asserts that there are at most finitely many conjugacy classes of minimum complexity.

Our overall plan, for other papers in this series, is to use the combinatorics of the foliation and associated structures to find ways to reduce the complexity if it is non-minimal. Some of that work is complete at this writing, and we describe it.

In a subsequent paper in this series [B-M, III] we find the finitely many conjugacy classes predicted by Theorem 2 in the first non-trivial case, i.e. links of braid index 3. The picture there is beautifully simple: links of braid index 3 have unique conjugacy classes of 3-braid representatives, with one class of exceptions: an infinite sequence of invertible links, each of which has two conjugacy classes of 3-braid representatives, differing only in the orientation of the braid and of the associated spanning surface.

The work in [B-M, III] is geometric, but as noted earlier it has an algebraic analogue. Motivated by the geometry in [B-M, II] and [BM, III], P. J. Xu sought and discovered a new solution in [X] to the conjugacy problem in the braid group $B_{3}$. Her solution was based directly upon the special properties of the pairs $[\mathbf{F}, \mathbf{H}]$ associated to minimum complexity. In the special case of $B_{3}$ these pairs are particularly simple. We have given them the name "Bennequin surfaces". 1

\footnotetext{
${ }^{1}$ Rudolph has also used the term "Bannequin surface" in [R], but his meaning differs from ours in [B-M, III].
} 
Her solution to the conjugacy problem in $B_{3}$ uses a non-standard set of generators for $B_{3}$, and is based upon shortest words in those generators. Xu's shortest words describe the sequence of half-twisted bands which define a Bannequin surface. They have to do with a type of generalized "crossing number," but if the braid index is 3 (rather than 2 or 1) her crossing numbers are not minimum crossing numbers in any projection. Her solution to the conjugacy problem actually enables her to find a spanning surface $\mathbf{F}$ of maximal Euler characteristic (however at this time one needs to begin with a 3-braid representative of $\mathscr{L}$ in order to do so).

In later papers in this series we will give further applications of the combinatorial data, the foliations and the complexity functions which are introduced here, using them to study some of the special problems which must be solved before our 3-braid results can be generalized to arbitrary braid index. The work in [B-M, IV] deals with the decomposition of a braid representative of a link type into braid representatives of its prime, irreducible summands. The manuscript [B-M, V] investigates the closed braid representatives of the unlink. In [B-M, VI] we characterize precisely the conditions under which a link admits infinitely many conjugacy classes of closed $n$-braid representatives, for arbitrary fixed $n$. The manuscript [B-M, VI] is the last of the preliminary steps which are needed before we can generalize our 3-braid results.

Acknowledgment. Special thanks go to Melissa Menasco and Joseph Birman, for support and encouragement during many visits of Birman to the Menasco home and Menasco to the Birman home. This work, and the work reported on in the references [B-M, II-VI], are part of a much larger study still in progress. We could not have attempted this work without their cooperation and strong support.

2. Basic machinery. Our work begins with an oriented link type $\mathscr{L}$ in oriented 3-space $\mathbb{R}^{3}$ or $S^{3}$, a closed braid representative $\mathbf{L}$ with braid axis $\mathbf{A}$ and a choice of fibration $\mathbf{H}$ of $\mathbb{R}^{3}-\mathbf{A}$. Our link $\mathbf{L}$ is the boundary of a family of $q \leq r$ disjointly embedded surfaces $\mathbf{F}=\left\{\mathbf{F}_{1}, \ldots, \mathbf{F}_{q}\right\}$ in $S^{3}$, which are assumed to be oriented so that the positive normal bundle to each component of $\mathbf{F}$ has the orientation induced by that on $\mathbf{L}=\partial \mathbf{F}$. Among all such surfaces $\mathbf{F}$ we assume that $\mathbf{F}$ has been chosen to be a family of pairwise disjoint surfaces of maximal Euler characteristic. For example, if $\mathscr{L}$ is the oriented $r$-component unlink, then $\mathbf{F}$ is a family of $r$ disjoint discs. On the other hand, if $\mathscr{L}$ is the Hopf link, the $\mathbf{F}$ will be a connected surface 

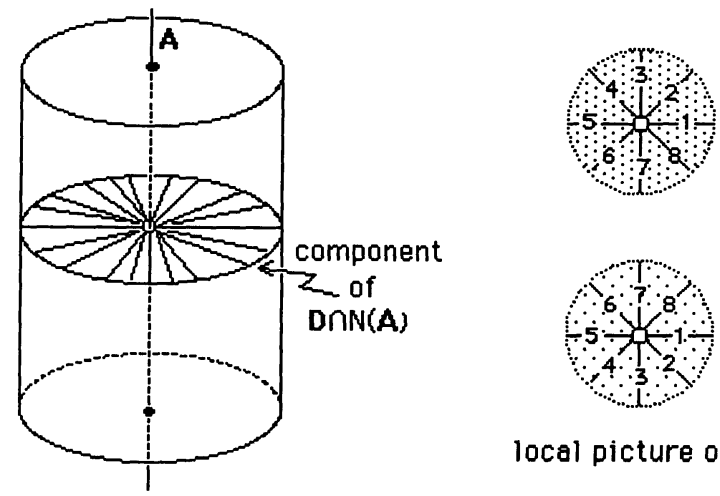

FIGURE 2

of genus 2. In general, $\mathbf{F}$ is not unique, so there is some arbitrariness in our choice, however once we have made a choice we will stick with it.

By general position, we may assume that:

(i) The intersections of $\mathbf{A}$ with $\mathbf{F}$ are finite in number and transverse.

(ii) There is a solid torus neighborhood $N(\mathbf{A})$ of $\mathbf{A}$ in $S^{3}-\mathbf{L}$ such that each component of $\mathbf{F} \cap N(\mathbf{A})$ is a disc. In particular, the foliation in each component of $\mathbf{F} \cap N(\mathbf{A})$ is the standard radial foliation. See Figure 2.

(iii) All but finitely many fibers $\mathbf{H}_{\theta}$ of $\mathbf{H}$ meet $\mathbf{F}$ transversally, and those which do not (the singular fibers) are each tangent to $\mathbf{F}$ at exactly one point in the interior of both $\mathbf{F}$ and $\mathbf{H}_{\theta}$. Moreover, the tangencies are local maxima or minima or saddle points.

With these restrictions we call $(\mathbf{F}, \mathbf{H})$ a nice pair. Figure 3 gives an example of a nice pair which represents the unknot, as a closed 2-braid.

Assume that $(\mathbf{F}, \mathbf{H})$ is a nice pair. Then the fibration $\mathbf{H}=\left\{\mathbf{H}_{\theta}\right.$; $\theta \in[0,2 \pi]\}$ induces a singular foliation of $\mathbf{F}$, the leaves being the components of $\left\{\mathbf{F} \cap \mathbf{H}_{\theta}, \theta \in[0,2 \pi]\right\}$. They are non-singular if $\mathbf{H}_{\theta}$ is not one of the finitely many singular fibers. Non-singular leaves can be either arcs or simple closed curves. (Note: we will prove, as Bennequin did, that we may assume there are no simple closed curves.) If they are arcs, their endpoints may be either on $\mathbf{L}=\partial \mathbf{F}$ or on $\mathbf{A}=\partial \mathbf{H}_{\theta}$ or both.

Let $\mathbf{A}$ and $\mathbf{A}^{\prime}$ be axes for an $n$-braid structure on $\mathscr{L}$, with braid representatives $\mathbf{L}$ and $\mathbf{L}^{\prime}$ and associated fibrations $\mathbf{H}=\left\{\mathbf{H}_{\theta} ; \theta \in\right.$ $[0,2 \pi]\}$ and $\mathbf{H}^{\prime}=\left\{\mathbf{H}_{\theta}^{\prime}: \theta \in[0,2 \pi]\right\}$. Assume that $(\mathbf{F}, \mathbf{H})$ and 




Figure 3

$\left(\mathbf{F}^{\prime}, \mathbf{H}^{\prime}\right)$ are nice pairs. We say that $(\mathbf{F}, \mathbf{H})$ is equivalent to $\left(\mathbf{F}^{\prime}, \mathbf{H}^{\prime}\right)$ if there is an isotopy $h_{t}$ of $\mathbb{R}^{3}, t \in[0,1]$, which takes the pair $(\mathbf{L}, \mathbf{A})$ to the pair $\left(\mathbf{L}^{\prime}, \mathbf{A}^{\prime}\right)$, in such a way that each $h_{t}(\mathbf{F}, \mathbf{H})$ is a nice pair. Thus, in particular, the isotopy $h_{t}$ takes:

1. A to $\mathbf{A}^{\prime}$ via a sequence of closed braid axes $h_{t}(\mathbf{A})$,

2. fibers of $\mathbf{H}$ to fibers of $\mathbf{H}^{\prime}$ via fibers $h_{t}(\mathbf{H})$,

3. $\mathbf{L}$ to $\mathbf{L}^{\prime}$ via closed braids $h_{t}(\mathbf{L})$ with axes $h_{t}(\mathbf{A})$,

4. $\mathbf{F}$ to $\mathbf{F}^{\prime}$ via a sequence of spanning surfaces $h_{t}(\mathbf{F})$.

Moreover, for each fixed $\theta \in[0,2 \pi]$, it restricts to an isotopy $h_{\theta, t}$ of $\left(\mathbf{F} \cap \mathbf{H}_{\theta}, \mathbf{F} \cap \partial \mathbf{H}_{\theta}\right)$ onto $\left(F^{\cdot} \cap \mathbf{H}_{\theta}^{\prime}, \mathbf{F}^{\cdot} \cap \partial \mathbf{H}_{\theta}^{\prime}\right)$, such that

$$
\begin{aligned}
& h_{\theta, 0}\left(\left(\mathbf{F} \cap \mathbf{H}_{\theta}, \mathbf{F} \cap \partial \mathbf{H}_{\theta}\right)\right)=\left(\mathbf{F} \cap \mathbf{H}_{\theta}, \mathbf{F} \cap \partial \mathbf{H}_{\theta}\right), \\
& h_{\theta, 1}\left(\left(\mathbf{F} \cap \mathbf{H}_{\theta}, \mathbf{F} \cap \partial \mathbf{H}_{\theta}\right)\right)=\left(\mathbf{F}^{\cdot} \cap \mathbf{H}_{\theta}^{\prime}, \mathbf{F}^{\cdot} \cap \partial \mathbf{H}_{\theta}^{\prime}\right) .
\end{aligned}
$$

Thus each non-singular leaf in the foliation of $\mathbf{F}$ is isotopic to a corresponding non-singular leaf in the foliation of $\mathbf{F}^{\prime}$, and similarly for singular leaves.

Let $[\mathbf{F}, \mathbf{H}]$ denote the equivalence class of $(\mathbf{F}, \mathbf{H})$. If $X$ is a finite set, let $|X|$ denote the number of elements in $X$. Our first entry in the complexity function is the braid index $\left|\mathbf{L} \cap \mathbf{H}_{\theta}\right|$ of $\mathbf{L}$. This integer is independent of $\theta$, because $L$ is a closed braid relative to $H$. The 
second entry is the number of points $|\mathbf{A} \cap \mathbf{F}|$ of intersection of $\mathbf{A}$ with $\mathbf{F}$. The third entry $|\mathbf{H} \cdot \mathbf{F}|$ is the number of tangencies between $\mathbf{F}$ and fibers of $\mathbf{H}$, or equivalently the number of singularities in the foliation of $\mathbf{F}$. The complexity $\mathbf{C}=C([\mathbf{F}, \mathbf{H}])$ is defined to be the triple:

$$
C([\mathbf{F}, \mathbf{H}])=\left(\left|\mathbf{L} \cap \mathbf{H}_{\theta}\right|,|\mathbf{A} \cap \mathbf{F}|,|\mathbf{H} \cdot \mathbf{F}|\right) .
$$

From our earlier observations, all representatives of an equivalence class $[\mathbf{F}, \mathbf{H}]$ have the same complexity. Thus $\mathbf{C}$ is well-defined on equivalence classes. Since conjugacy classes in $B_{n}$ are determined by equivalence classes $[\mathbf{F}, \mathbf{H}]$ it follows that complexity is well defined on conjugacy classes in $B_{n}$.

We assign the standard lexicographic ordering to equivalence classes $[\mathbf{F}, \mathbf{H}]$ associated to a single link type $\mathscr{L}$, relative to this complexity function. This assigns a partial order to the conjugacy classes in $B_{\infty}$ which represent $\mathscr{L}$.

We begin to study the foliation of F. A singular leaf in the foliation will be one which contains a point of tangency with a fiber of $\mathbf{H}$. All other leaves are non-singular. Note that it follows from property (iii) of nice pairs that:

(a) Each non-singular leaf is an arc or a circle.

(b) A singular fiber contains exactly one singular point.

(c) Each singular point is either a center or a saddle.

In principle, the non-singular leaves of the foliation which are arcs could have their endpoints on $\mathbf{A}=\partial \mathbf{H}_{\theta}$ or on $\mathbf{L}=\partial \mathbf{F}$. However, we claim:

LEMMA 1. An arc in $\mathbf{H}_{\theta} \cap \mathbf{F}, \mathbf{H}_{\theta}$ non-singular, cannot have both of its endpoints on $\mathbf{L}$.

Proof of Lemma 1. Suppose that $\mu$ were such an arc. Let $N(\mu)$ be a neighborhood of $\mu$ on $\mathbf{F}$. Since $\mathbf{F}$ is oriented, and the orientation on $\mathbf{L}=\partial \mathbf{F}$ is consistent with the orientation on $\mathbf{F}$, it follows that $\mathbf{L}$ must be oriented in opposite directions at the two ends of $N(\mu)$. (See Figure 4a.) The picture on $\mathbf{H}_{\theta}$ must then be as in Figure 4b. However $L$ is a closed $n$-braid, so $L$ meets each fiber $\mathbf{H}_{\theta}$ in exactly $n$ transverse, coherently oriented intersections, so this can't happen.

In view of Lemma 1, the leaves in the foliation of $\mathbf{F}$ are either simple closed curves or arcs, where the arcs can be subdivided into 


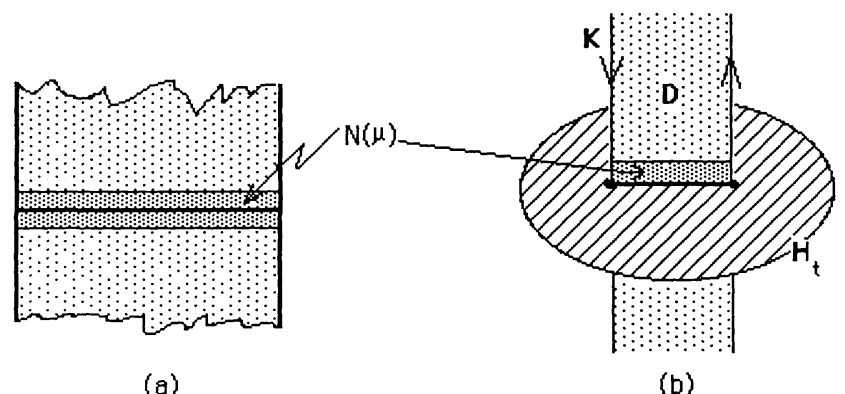

FIGURE 4

two types:

a-arcs: arcs which have one endpoint on $\mathbf{A}$ and one on $\mathbf{L}-\partial \mathbf{F}$.

b-arcs: arcs which have both endpoints on $\mathbf{A}$.

We now investigate simple closed curves (scc's) in $\mathbf{F} \cap \mathbf{H}$. We say that $\mathbf{F}$ has scc's if there exists a singular fiber $\mathbf{H}_{\theta}$ such that a component of $\mathbf{F} \cap \mathbf{H}_{\theta}$ is a scc. Otherwise, $\mathbf{F}$ has no scc's.

Lemma 2. Assume that F satisfies (i)-(iii) and has scc's. Then there exists $\mathbf{F}^{*}$, with $\partial \mathbf{F}^{*}=\partial \mathbf{F}$, such that $\mathbf{F}^{\cdot}$ also satisfies (i)-(iii), and in addition has no scc's; moreover, $C\left(\left[\mathbf{F}^{*}, \mathbf{H}\right]\right) \leq C([\mathbf{F}, \mathbf{H}])$. If in addition $S^{3}-\mathbf{L}$ is reducible (every 2-sphere bounds a 3-ball) then $F^{\cdot}$ is isotopic to $\mathbf{F}$ via an isotopy which leaves $\mathbf{L}=\partial \mathbf{F}$ fixed pointwise.

Proof of Lemma 2. Our proof, below, is essentially the argument given on pages 106-107 of [Be]. Our surface $\mathbf{F}^{\prime}$ is called a "Markov surface" in those papers, and the proof we now give establishes the key property of such surfaces. We include it to make this series of papers self-contained and also because we need more detail than is given in [Be], and a more systematic approach, in order to be able to extend our results to more general types of surfaces.

Suppose that there is a scc $\alpha(\theta)$ in $\mathbf{F}_{i} \cap \mathbf{H}_{\theta}$ for some non-singular $\mathbf{H}_{\theta}$. The fact that $\mathbf{F}_{i}$ is pierced non-trivially by the braid axis $\mathbf{A}$ implies (by property (iii)) that all of $\mathbf{F}_{i}$ cannot be foliated by scc's, so if we follow the sequence of $\operatorname{arcs} \alpha(\theta)$ as $\theta$ increases or decreases we will eventually arrive at a singularity $s_{0}$.

By property (iii), the singularity is either a center or a saddle point. However, if it is a center, then if we reverse the sense of change in $\theta$ and follow $\alpha(\theta)$ in the opposite direction we will necessarily arrive at a second singularity, and that one cannot also be a center because F is not a 2-sphere, so we can assume that our singularity is a saddle 
point. Let $\theta_{0}$ denote the value of $\theta$ at the singularity, let $s_{0}$ be the singular point, and let $\Delta$ be the disc which $\alpha\left(\theta_{0}\right)$ bounds on $\mathbf{H}_{\theta_{0}}$.

By property (iii) the point $s_{0}$ is the only singular point on the fiber at $\theta_{0}$, so the singularity is necessarily a homoclinic point. Figure 5 illustrates such a homoclinic point-a singularity which is formed when a generic leaf has a saddle point singularity with itself. Regarding $\alpha\left(\theta_{0}\right)$ as a curve on the fiber $\mathbf{H}_{\theta_{0}}$, there are two possible situations, shown as cases 1 and 2 . However, by Lemma 1 every component of $\mathbf{F} \cap \mathbf{H}_{\theta_{0}}$ which is in $\Delta$ must be a simple closed curve, and from this it follows that in case 2 the dotted strands necessarily join up as shown inside $\Delta$, so in fact if the second case occurs then the first does too. Thus without loss of generality we may assume that we are in the situation of Figure 5, case 1.
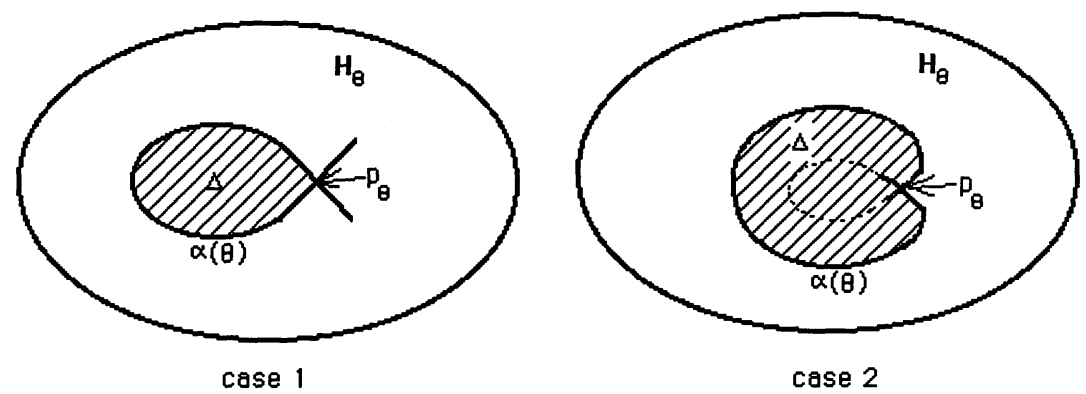

Figure 5

It may happen that the interior of the disc $\Delta$ in Figure 5 , case 1 , has empty intersection with $\mathbf{F}$. If so, we can surger $\mathbf{F}$ along $\alpha(\theta)$ as in Figure 6. Let $\mathbf{D}^{\sim}$ be the surgered surface. Now observe that the surgered surface must have one more component than did $\mathbf{F}$; also one of the new components, say $\mathbf{F}^{\sim}(1)$, must be a topological 2-sphere, for if not our surgery would have increased the Euler characteristic. We can then discard $\mathbf{F}^{\sim}(1)$ to produce a new surface $\mathbf{F}^{\#}$ with $\partial \mathbf{F}^{\#}=\partial \mathbf{F}$. A small isotopy of $\mathbf{F}^{\#}$ (Figure 6 again) produces a surface $\mathbf{F}^{\prime}$ which is transverse to $\mathbf{H}$ in the region which we just modified. Its foliation has fewer homoclinic points than the foliation of $\mathbf{F}$. Induction on the number of such points completes the proof.

It remains to consider the case when the interior of $\Delta$ intersects $F$. See Figure 7. By property (iii) there are no singularities of $\mathbf{F} \cap \mathbf{H}_{\theta_{0}}$ in the interior of $\Delta$. By Lemma 1 each component of $F \cap i n t \Delta$ must then be a scc. Let $c$ be an innermost such scc. Then we can surger F along $c$, as in Figure 7. A small isotopy of the surgered surface 

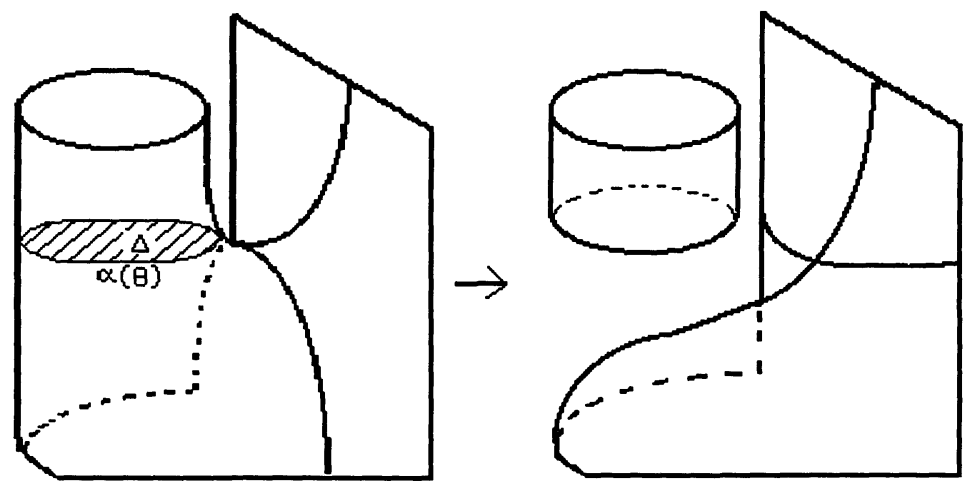

FIGURE 6

suffices to restore transversality with fibers of $\mathbf{H}$. Note that we can do this isotopy so that at most two new tangencies with fibers of $\mathbf{H}$ are produced by the surgery, both being local maxima or minima. Let $\mathbf{F}^{\sim}$ be the resulting surface. We now observe that as before our surgery necessarily produces a closed component $\mathbf{F}^{\sim}(1)$ which is homeomorphic to $S^{2}$. Set $\mathbf{F}^{\#}=\mathbf{F}-\mathbf{F}^{\sim}(1)$. The 2-sphere $\mathbf{F}^{\sim}(1)$ must be tangent to $H$ at at least two points, so that when we throw away $\mathbf{F}^{\sim}(1)$ we eliminate at least two centers. Thus, even though we have introduced an extra center in the part of $\mathbf{F}^{\sim}$ which we retain, the total number of singularities of each type in the foliation of $\mathbf{F}^{\#}$ cannot be more than that in the foliation of $\mathbf{F}$.

After a finite number of repetitions of the surgeries depicted in Figures 4 and 5 we will obtain a surface $F^{\prime}$ which satisfies (i)-(iii) as before; also $C\left(\left[\mathbf{F}^{\prime}, \mathbf{H}\right]\right) \leq C([\mathbf{F}, \mathbf{H}])$. Even more there are no homoclinic singular points in the foliation of $\mathbf{F}^{\prime}$, because the surgeries
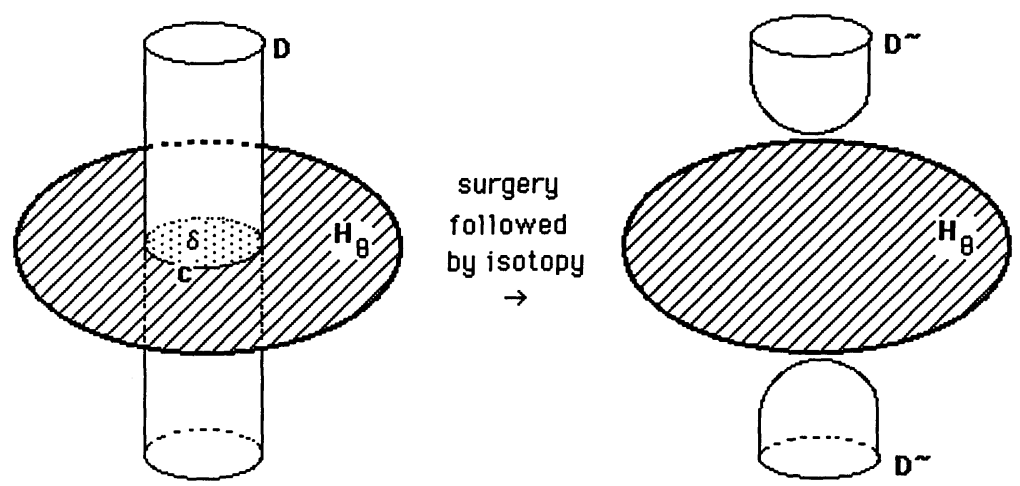

FIGURE 7 
depicted in Figure 6 eliminated them. Since the existence of a scc in the foliation of $\mathbf{F}^{\prime}$ implies the existence of a homoclinic point, we conclude that $\mathbf{F}^{\prime}$ has no scc's.

Our final observation is that if $S^{3}-\mathbf{L}$ is irreducible, then each time we do a surgery which splits off one of the 2-spheres we called $\mathbf{F}^{\sim}(1)$, that 2-sphere will bound a 3-ball $\mathbf{B}$. It follows that the surgery which produced $\mathbf{F}^{\sim}$ could have been replaced by an isotopy of $\mathbf{F}$ through B. The proof of Lemma 2 is complete.

REMARK 1. In future papers, on related work, we will need to use Lemma 2 in other settings, with slightly different hypotheses. We therefore take this opportunity to note that all of our standardizations in the geometry, and also our definition of the complexity function, and also the statement and proof of Lemma 2, go through without any essential changes if we make the following modifications in the hypotheses:

LEMMA $2^{*}$. Augment $\mathbf{F}$ by taking its disjoint union with a collection $\Omega$ of pairwise disjoint essential discs which are properly embedded in the 3-manifold $\mathbf{M}$ obtained by splitting $S^{3}$ along $\mathbf{F}$. Assume that $\mathbf{F}$ and also $\Omega$ satisfy (i)-(iii) and has scc's. Modify the complexity function to $C([\mathbf{F} \cup \Omega, \mathbf{H}])=(n,|\mathbf{A} \cap \mathbf{F}|+|\mathbf{A} \cap \Omega|,|\mathbf{H} \cdot \mathbf{F}|+|\mathbf{H} \cdot \Omega|)$. Then there exists $\mathbf{F}^{\prime}$ and $\Omega^{\prime}$, with $\partial \mathbf{F}^{\prime}=\partial \mathbf{F}, \partial \Omega^{\prime}=\partial \Omega$, such that $\mathbf{F}^{\prime}$ and $\Omega^{\prime}$ satisfy (i)-(iii) and have no scc's, and also $C\left(\left[\mathbf{F}^{\prime} \cup \Omega^{\prime}, \mathbf{H}\right]\right) \leq C([\mathbf{F} \cup \Omega, \mathbf{H}])$.

LEMMA $2^{* *}$. If $\mathscr{L}$ is a reducible link type, and $\mathbf{X}$ is a splitting 2sphere, or if $\mathscr{L}$ is composite and $\mathbf{Y}$ is a 2-sphere which realizes the connected sum decomposition, we may eliminate scc's from the foliation of $\mathbf{X}$ and of $\mathbf{Y}$ just as we eliminated them from the foliation of $\mathbf{F}$. See [B-M, IV] for detailed studies of these two situations. Also, see [Mo2] for an earlier treatment of closed braids which are composite.

3. Combinatorial data and the finiteness theorem. We will show in this section that the fibration $\mathbf{H}$ determines (via the induced foliation on each component of F) a finite set of combinatorial data. Theorem 1 asserts that this data determines $F$ as an embedded surface and $\mathbf{L}=\partial \mathbf{F}$ as a closed braid. As a corollary, we will prove that there are at most finitely many conjugacy classes in $B_{\infty}$ which represent a given link type and have minimum complexity.

We begin our work by investigating the singularities in the foliation of $\mathbf{F}$. By our hypotheses on $\mathbf{F}$ and $\mathbf{H}$ the components of 
$\mathbf{F} \cap \mathbf{H}_{\theta}$, for each non-singular $\mathbf{H}_{\theta}$, will be a union of a-arcs and barcs. If $a_{1}, \ldots, a_{m}, b_{1}, \ldots, b_{\mu}$ are the a-arcs and $\mathbf{b}$-arcs in some non-singular $\mathbf{H}_{\theta}$ then each arc can be characterized by the label of its endpoints. We label the endpoints which are on $\mathbf{A}$ as $p_{1}, \ldots, p_{m+2 \mu}$, in the (cyclic) order in which they occur on $\mathbf{A}$. We investigate the foliation of $\mathbf{F}$ near its singular points. For later use, we label the singularities $s_{1}, \ldots, s_{k}$, in the order in which they occur in the fibration.

Since we have two types of arcs, a-arcs and b-arcs, there are at most three types of singularities:

type aa: an a-arc surgered with an a-arc,

type $a b$ : an a-arc surgered with a b-arc,

type $b b$ : a b-arc surgered with a b-arc.

By hypothesis the singular points of the foliation occur at the interiors of the arcs which are involved. The left column in Figure 8 shows a sequence of leaves in $\mathbf{F}$ before and after a type $a a$ singularity, where since $\mathbf{F}$ is an oriented surface we can assume we are looking at its positive side $\mathrm{F}^{+}$. The top left picture in Figure 9 shows the induced foliation on $\mathbf{F}^{+}$. The arrows indicate the direction of increasing $t$. We have extended the singular leaves to their endpoints, two of which are on $\mathbf{A}$ and two of which are on $\mathbf{L}=\partial \mathbf{F}$.

The middle column in Figure 8 shows a sequence of leaves in $\mathbf{F}^{+}$ just before and after a type $a b$ singularity. The corresponding picture in the top row in Figure 9 shows the induced foliation on $\mathbf{F}^{+}$. In this case, when we extend the singular leaves to their endpoints, we see that three of the endpoints are on $\mathbf{A}$ and one is on $\mathbf{L}$. Finally, the right column in Figure 8 shows a sequence of leaves of $\mathbf{F}^{+}$before and after a type $b b$ singularity, and the corresponding picture in Figure 9 shows the induced foliation on $\mathbf{F}^{+}$. In this case all four endpoints of the singular leaves are on $\mathbf{A}$.

Note that the complement of the set of all singular leaves is a collection of regions which are foliated without singularities. Since there are exactly two types of arcs, there are also two types of regions: those which are foliated entirely by a-arcs, and those which are foliated entirely by b-arcs. These are depicted in the bottom row in Figure 9.

Let $\mathbf{H}\left(\theta_{1}\right), \ldots, \mathbf{H}\left(\theta_{k}\right)$ be the cyclically ordered array of singular fibers of $\mathbf{H}$, i.e. of fibers which are tangent to $\mathbf{F}$, each $\mathbf{H}\left(\theta_{z}\right)$ containing its set of singular leaves in the foliation of $\mathbf{F}$. Call this the cycle of fibers. In order to highlight our next result, we note that it is well known that every closed braid is determined by a set of combinatorial data, e.g. by its description as a word in generators of the braid group. We can say more. 


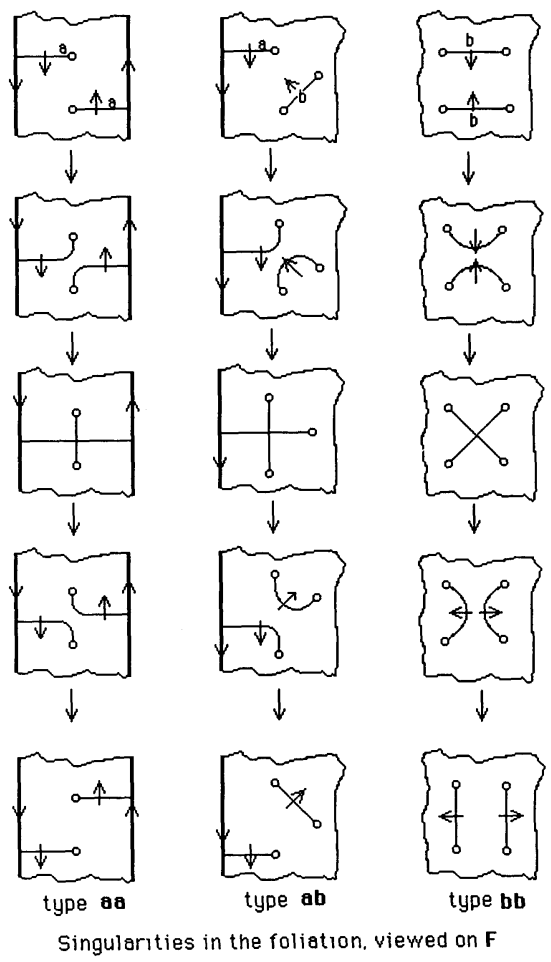

FIGURE 8

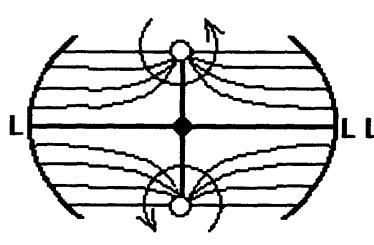

type aa

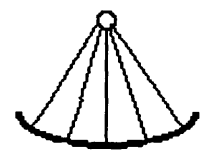

Foliated regions in the complement of the singular leaves

FIGURE 9

Theorem 1. Fix a fibration $\mathbf{H}$ of $S^{3}-\mathbf{A}$. Choose a surface $\mathbf{F}$ of maximum Euler characteristic with $\partial \mathbf{F}=\mathbf{L}$. Then a finite set of combinatorial data suffices to determine $\mathbf{F}$ as an embedded surface, with $(\mathbf{F}, \mathbf{H})$ a nice pair and $\mathbf{L}$ a closed braid relative to $\mathbf{A}$. 
Proof. We associate to our foliated surface $\mathbf{F}$ a finite set of combinatorial data which is determined by the cycle of fibers and which will be shown to determine the embedding of $\mathbf{F}$ in $S^{3}$. First, recall that $p_{1}, \ldots, p_{m+2 \mu}$ are the points where the axis $\mathbf{A}$ pierces the oriented surface $\mathbf{F}$, labeled to correspond to their natural cyclic order on A. Associated to each $p_{x}$ a sign, which is taken to be + or according as the oriented axis $\mathbf{A}$ pierces $\mathbf{F}$ from the positive to the negative side, or the negative to the positive side at $x$. To clarify the concept of sign, we return momentarily to Figure 2, and study the detail, which shows how things look on $\mathbf{F}$ near a point where $\mathbf{A}$ pierces $\mathbf{F}$. We have shown 8 leaves in the foliation, labeling them as they might occur in a cycle of 8 fibers around $\mathbf{A}$, the number "i" indicating time " $t_{i}$." There are two cases, depending upon whether $\mathbf{A}$ pierces $\mathbf{F}$ from the positive or negative side. Let $\mathbf{P}$ denote the cycle array $\mathbf{P}=\left(\xi_{1}, \xi_{2}, \ldots, \xi_{m+2 \mu}\right)$ where $\xi_{i}= \pm$ is the sign of $p_{i}$.

If $s_{z}$ is a singular point of the foliation, define the sign of $s_{z}$ to be + or - according as the outward drawn normal to $\mathbf{F}$ at $s_{z}$ points in the direction of increasing or decreasing $\theta$. Next, note that there are exactly four singular leaves of the foliation of $\mathbf{F}$ which meet at each singular point $s_{z}$ (Figure 9). Assuming that we are looking at $\mathbf{H}\left(\theta_{z}\right)$ in the direction of increasing polar angle $\theta$, the leaves have a natural cyclic order. If we follow the four leaves out to their four endpoints we see that two, three or four of them have their endpoints at one of the pierce-points, according as $s_{z}$ has type $a, a b$ or $b b$, and then associate to $s_{z}$ the unordered 2-tuple $\left(p_{i}, p_{j}\right)$, ordered 3tuple $\left(p_{i}, p_{j}, p_{k}\right)$, or cyclically ordered 4-tuple $\left(p_{i}, p_{j}, p_{k}, p_{w}\right)$ of A-endpoints of its singular leaves, according as $s_{z}$ has type $a a, a b$ or $b b$. (Our convention, for type $a b$, is that the first pierce-point is the one which follows $\partial F$ in the clockwise order.) Call this 2, 3 or 4-tuple $+\mathbf{T}_{z}$ or $-\mathbf{T}_{z}$, where the sign denotes the sign of the singularity. Let $\mathbf{T}$ denote the cyclic array $\mathbf{T}=\left( \pm \mathbf{T}_{1}, \pm \mathbf{T}_{2}, \ldots, \pm \mathbf{T}_{q}\right)$, where each $\mathbf{T}_{z}$ is a tuple $\left(p_{i}, p_{j}\right),\left(p_{i}, p_{j}, p_{k}\right)$, or $\left(p_{i}, p_{j}, p_{k}, p_{w}\right)$ of points from the cyclically ordered set $p_{1}, \ldots, p_{m+2 \mu}$. The combinatorial symbol associated to the tiling of $\mathbf{F}$ is the pair $(\mathbf{P}, \mathbf{T})$.

To reconstruct $\mathbf{F}$ as an embedded surface from the data in $(\mathbf{P}, \mathbf{T})$ we begin with $\mathbf{A}$ as the $z$-axis in 3-space and $\mathbf{H}$ as the standard fibration of $R^{3}-\mathbf{A}$ by half-planes with polar angle $\theta \in[0,2 \pi]$. Choose $m+2 \mu$ points on $\mathbf{A}$, and declare them to be $p_{1}, \ldots, p_{m+2 \mu}$. Up to a homeomorphism of $S^{3}$ which fixes each fiber setwise, it will not matter exactly where the points are located on $\mathbf{A}$, as long as their cyclic order is correct. We now attach to each $p_{x}$ a small disc $\delta_{x}$ 
which is the component of $\mathbf{F} \cap \mathbf{N}(\mathbf{A})$ which contains $p_{x}$. This disc has the standard radial foliation. The sign of $p_{x}$ is positive or negative according as the orientation on $\mathbf{A}$ agrees or disagrees with that on the normal bundle to $\mathbf{F}$ at $p_{x}$. This is determined by the data on $\mathbf{P}$, which therefore tells us whether $\mathbf{A}$ pierces $\delta_{x}$ from the negative or positive side.

We next select, arbitrarily, $q$ distinct fibers, ordered by increasing polar coordinate $\theta$ in $[0,2 \pi]$, and a point on each, and declare these points to be the singular points $s_{1}, \ldots, s_{q}$ of the foliation of F. Call these fibers $\mathbf{H}\left(\theta_{1}\right), \ldots, \mathbf{H}\left(\theta_{q}\right)$. It will not matter which fibers we choose, as long as we keep their order unchanged, because there is always a reparametrization of the intervals $[0,2 \pi]$ which will take any one choice of singular fibers to any other. It also will not matter where we place the points on the fibers, because again there is a homeomorphism of $S^{3}$ which fixes each fiber and takes any one point on a single fiber to any other point on the same fiber.

Each singular point $s_{z}$ is a saddle-point tangency of $\mathbf{F}$ with the fiber $\mathbf{H}\left(\theta_{z}\right)$, so that $\mathbf{F} \cap \mathbf{H}\left(\theta_{z}\right)$ will be a union of four arcs which go out from $s_{z}$ like the spokes on a wheel. See Figure 10. If the singularity $s_{z}$ is type $a b$ (or $b b$ ), we select 3 (or all 4) of the arcs which meet at $s_{z}$, order them clockwise on $\mathbf{H}\left(\theta_{z}\right)$, viewed in the direction of increasing $\theta$, and extend them to long arcs in $\mathbf{H}\left(\theta_{z}\right)$ which end at the 3 or 4 pierce-points in the tuple $\mathbf{T}_{z}$. If $s_{z}$ is type $a a$, the order won't matter, however now we select a pair of opposite arcs and extend them in $\mathbf{H}\left(\theta_{z}\right)$ to the two pierce points in the tuple $\mathbf{T}_{z}$. Note that in general there will be several singular arcs meeting at each pierce-point $p_{x}$. They will coincide with leaves in the radial foliation of the component of $\mathbf{F} \cap \mathbf{N}(\mathbf{A})$ at $p_{x}$. Their order around $p_{x}$ is determined by the order of the singular points $s_{1}, \ldots, s_{k}$ in the fibration. In the case of type $a a$ (or $a b$ ) tiles, there will be 2 (or 1) singular leaves which are unaccounted for. They can be made arbitrarily long. Their endpoints will be the points where $\mathbf{L}$ pierces the singular fiber $\mathbf{H}\left(\theta_{z}\right)$.

We have now embedded a spine $\mathbf{S}$ of $\mathbf{F}$ in $S^{3}$. Let $\mathbf{U}$ be a neighborhood of $\mathbf{S}$ in $\mathbf{F}$. The next step is to embed $\mathbf{U}$ in $S^{3}$. That's easy, because all of the singular points are in $\mathbf{S}$, so that $\mathbf{U}-\mathbf{S}$ is transverse to every fiber. Moreover, knowing the sign of the singularity, we know which way to orient $\mathbf{U}$ relative to the direction of increasing $\theta$.

Recall now that each region in the complement of $\mathbf{S}$ in $\mathbf{F}$ is one of the two types shown in Figure 9. The first type of region is foliated 


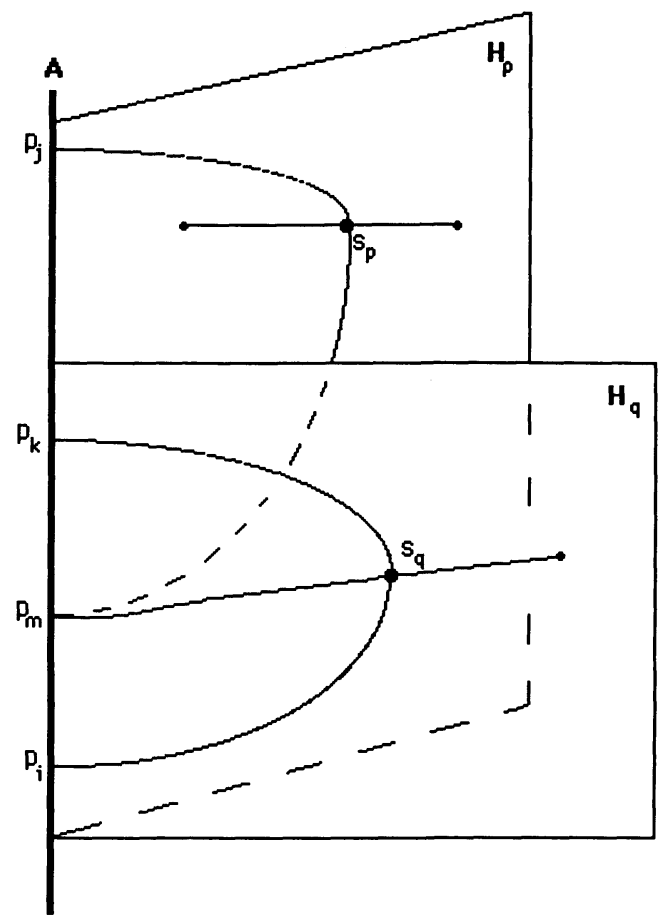

FiguRe 10

entirely by a-arcs, and the second is foliated entirely by b-arcs. Since each of these regions has non-trivial intersection with the disc components of $\mathbf{F} \cap \mathbf{N}(\mathbf{A})$, we can start to attach them at the points where they meet $\mathbf{F} \cap \mathbf{N}(\mathbf{A})$. The foliation in each region will be a family of level sets for the embedding, so there is a unique way to attach them. When we are finished attaching them we will have embedded $\mathbf{F}$ and so also $\mathbf{L}$ in $S^{3}$. This completes the proof.

THEOREM 2. There are at most finitely many conjugacy classes in $B_{\infty}$ which represent a fixed link type $\mathscr{L}$ and which have minimum complexity. Also, for each fixed value $n$ of the number of braid strands, there are at most finitely many conjugacy classes in $B_{n}$ which represent a fixed link type $\mathscr{L}$ and which have minimum complexity.

Proof. The complexity of $[\mathbf{F}, \mathbf{H}]$ is the triple $(n, m+2 \mu, q)$, where $n$ is the number of braid strands in $\mathbf{L}=\partial \mathbf{F}, m+2 \mu$ is the number of pierce-points and $q$ is the number of singularities in the foliation of $\mathbf{F}$. There are $2^{m+2 \mu}$ members of $\mathbf{P}$. Since $\mathbf{T}$ has $q$ members, and each is a 2,3 or 4-tuple of points from a set of cardinality $m+2 \mu$, 
it is then immediate that there are at most finitely many distinct sets of combinatorial data of complexity $(n, m+2 \mu, q)$. By Theorem 1 , each pair $(\mathbf{P}, \mathbf{T})$ determines $\mathbf{F}$ as a surface which is embedded in 3-space relative to $\mathbf{H}$. Thus there are at most finitely many conjugacy classes of minimal complexity.

REMARK 2. In [B-M, VI] we will show that the combinatorial data associated to the aa and ab singularities (but not the bb singularities) suffices to determine $\mathbf{L}$ as an embedded curve (but not $\mathbf{F}$ as an embedded surface). Thus, in principle, one should be able to recover an explicit word in the standard elementary braids which generate the $n$-string braid group from this combinatorial data. It seems to be an interesting problem to develop an algorithm to do so.

REMARK 3. The combinatorial data relates to features in the foliation of F. In Remark 1 above we noted that the work we had done up to and including Lemma 2 applies equally well if we replace $\mathbf{F}$ by a splitting 2-sphere $\mathbf{X}$ or by a collection $\Omega$ of essential discs which are properly embedded in $S^{3}-\mathbf{F}$, or by the disjoint union of $\mathbf{F}$ with either $\mathbf{X}$ and or $\Omega$. The same is true for the theorem.

We can say a little more. The leaves of the foliation of $\mathbf{F}$ are in $\mathbf{F} \cap \mathbf{H}_{\theta}$, so they can be regarded as subsets of either $\mathbf{F}$ or $\mathbf{H}_{\theta}$. Thus it is obvious that there is an equivalent set of combinatorial data which can be viewed in a judicious choice of a finite set of fibers in the collection $\left\{\mathbf{H}_{\theta} ; \theta \in[0,2 \pi]\right\}$. The appropriate data will be described when we need it, in other papers in this series.

\section{REFERENCES}

[A1] J. W. Alexander, A lemma on systems of knotted curves, Proc. Nat. Acad. Sci., U.S.A., 9 (1923), 93-95.

[Ar] E. Artin, Theorie der Zopfe, Hamburg Abh., 4 (1925), 47-72.

[Be] Daniel Bennequin, Entrelacements et equations de Pfaff, Astérisque, 107108 (1983), 87-161.

[Bi] Joan S. Birman, Braids, links and mapping class groups, Annals of Math. Studies \# 82, Princeton University Press, 1974.

[B-M] J. Birman and W. Menasco, A calculus for links in the 3-sphere, Proc. of 1990 Osaka Conference on "Knots", to appear.

[B-M, II] _ Studying links via closed braids II: On a theorem of Bennequin, Topology Appl., 40 (1991), 71-82.

[B-M, III] _ Studying links via closed braids III: Classifying links which are closed 3-braids, Pacific J. Math., to appear.

[B-M, IV] _ Studying links via closed braids IV: Split and composite links, Invent. Math., 102 (1990), 115-139. 
[B-M, V] J. Birman and W. Menasco, Studying links via closed braids V: The unlink, Trans. Amer. Math. Soc., 329 (1992), 585-606.

[B-M, VI] _ Studying links via closed braids VI: A non-finiteness theorem, Pacific J. Math., to appear.

[G] F. Garside, The braid groups and other groups, Quarterly J. Math. Oxford, 20, No. 78, 1968, pp. 235-254.

[Ma] A. A. Markov, Uber die freie aquivalenz der geschlossenen Zopfe, Recueil de la Soc. math. de Moscou, 43 (1936), 73-78.

[Mo1] H. Morton, Infinitely many fibered knots with the same Alexander polynomial, Topology, 17 (1978), 101-104.

[Mo2] _ Closed braids which are not prime knots, Math. Proc. Camb. Phil. Soc., 86 (1979), 421-426.

[Mo3] __ Exchangeable braids, London Math. Soc. Lecture notes 95, Ẽow dimensional topology ed. Fenn, 86-105.

[R] L. Rudolph, Special positions for surfaces bounded by closed braids, Rev. Mat. Iberoamericana, 1 no. 3 (1985), 93-133.

[Ta] P. G. Tait, On Knots, in Collected Scientific Papers of P. G. Tait, Camb. Univ. Press, London, 1898, 273-347.

[Th] W. Thurston, On the geometry and dynamics of diffeomorphisms of surfaces, Bull. Amer. Math. Soc. (New Series) 19, No. 2 (October 1988), 417-432.

[X] P. Xu, Ph.D. thesis.

Received March 5, 1990 and in revised form May 29, 1991. The first author was partially supported by NSF Grant DMS-88-05672 and the second author was partially supported by NSF Grant DMS-90-02673. Part of this work was done when the first author was a member of the Institute for Advanced Study. Partial support is gratefully acknowledged.

COLUMbia UNIVERSITY

NEW YORK, NY 10027

AND

SUNY AT BUFFALO

BUFFALO, NY 14222 


\section{PACIFIC JOURNAL OF MATHEMATICS EDITORS}

V. S. VARAdARAJAN

(Managing Editor)

University of California

Los Angeles, CA 90024-1555

Herbert Clemens

University of Utah

Salt Lake City, UT 84112

F. Michael CHRIST

University of California

Los Angeles, CA 90024-1555

ThOMAs ENRIGHT

University of California, San Diego

La Jolla, CA 92093
Nicholas ERCOLANI

University of Arizona

Tucson, AZ 85721

R. FINN

Stanford University

Stanford, CA 94305

VAUGHAN F. R. JoNES

University of California

Berkeley, CA 94720

SteVen KercKhofF

Stanford University

Stanford, CA 94305
C. C. MOORE

University of California

Berkeley, CA 94720

MARTIN SCHARLEMANN

University of California

Santa Barbara, CA 93106

HAROLD STARK

University of California, San Diego

La Jolla, CA 92093

\section{ASSOCIATE EDITORS}
R. Arens
E. F. BeCKenBACH
B. H. NeumanN
F. WolF
(1904-1989)
K. YoshidA (1906-1982)

TIONS

UNIVERSITY OF ARIZONA

UNIVERSITY OF BRITISH COLUMBIA

UNIVERSITY OF OREGON

UNIVERSITY OF SOUTHERN CALIFORNIA

CALIFORNIA INSTITUTE OF TECHNOLOGY

UNIVERSITY OF CALIFORNIA

MONTANA STATE UNIVERSITY

STANFORD UNIVERSITY

UNIVERSITY OF HAWAII

UNIVERSITY OF NEVADA, RENO

UNIVERSITY OF TOKYO

NEW MEXICO STATE UNIVERSITY

UNIVERSITY OF UTAH

OREGON STATE UNIVERSITY

WASHINGTON STATE UNIVERSITY

UNIVERSITY OF WASHINGTON 


\section{Pacific Journal of Mathematics}

Vol. 154, No. $1 \quad$ May, 1992

Richard Arens, Pseudo regular elements in a normed ring $\ldots \ldots \ldots \ldots \ldots 1$

Joan Birman and William W. Menasco, Studying links via closed braids.

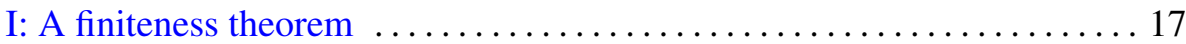

Etsurō Date, Michio Jimbo, Kei Miki and Tetsuji Miwa, Braid group

representations arising from the generalized chiral Potts models ....... 37

Toshihiro Hamachi, A measure theoretical proof of the Connes-Woods

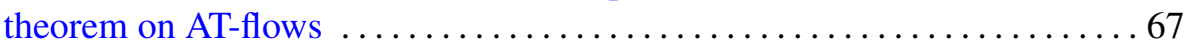

Allen E. Hatcher and Ulrich Oertel, Affine lamination spaces for surfaces ....................................... 87

David Joyner, Simple local trace formulas for unramified $p$-adic groups $\ldots .103$

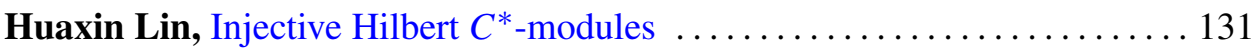

John Marafino, The boundary of a simply connected domain at an inner

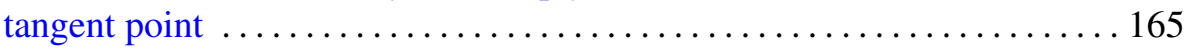

Gonzalo Riera and Rubi Rodriguez, The period matrix of Bring's curve . . 179 